\title{
SEM CHARACTERIZATION OF MULTILAYER STRUCTURES
}

\author{
V.V. Aristov, N.N. Dryomova, V.A. Kireev, I.I. Razgonov \\ AND E.B. YaKIMOV
}

Institute of Microelectronics Technology and High Purity Materials Russian Academy of Sciences, 142432, Chernogolovka, Moscow district, Russia

\author{
Dedicated to Professor Dr. Julian Auleytner \\ on the occasion of his 70th birthday
}

(Received July 23, 1992)

\begin{abstract}
The possibilities of non-destructive multilayer structure characterization using the backscattering electron and modulated cathodoluminescence modes of the SEM have been discussed. It is shown that these techniques allow one to measure the parameters of thin layers of the thickness of about $10 \mathrm{~nm}$.
\end{abstract}

PACS numbers: 79.20.Fv, 68.35.Dv

\section{Introduction}

Multilayer structures are now widely used in numerous fields of technology, therefore the development of techniques for non-destructive characterization of these structures is of great importance. The thickness of layers in multilayer structures can range from $1 \mathrm{~nm}$ to some $\mu \mathrm{m}$, thus in order to measure their parameters it is necessary to develop different techniques with an appropriate spatial resolution. Very promising for these purposes are the methods based on the scanning electron microscopy (SEM) measurements. In principle, the SEM techniques allow one to achieve a high spatial resolution but usually the resolution degrades due to large enough dimensions of the signal formation region. Therefore, in order to realize the high spatial resolution, some special methods or image processing procedures should be developed [1-3]. In the case of a non-destructive characterization of planar multilayer structures the electron beam is directed perpendicular to the structure plane while the main parameters change as a function of depth. Therefore a third variable for the reconstruction of the parameter distribution should be used. As the third variable an electron beam energy $E_{\mathrm{b}}$ is usually used, which determines the primary electron penetration depth, i.e. a set of images with $E_{\mathrm{b}}$ as a parameter is obtained and the property distribution is reconstructed on the base of such measurements. 
In the present paper the possibilities of two such techniques for the multilayer structure characterization using the measurements of the signal dependence on the primary electron energy are discussed. The first one is based on the backscattering electron (BSE) coefficient measurements, the second one is the modulated cathodoluminescence (MCL) technique. It has been shown that the BSE signal from multilayer structures has maxima in the $E_{\mathrm{b}}$ dependence. The number of maxima is equal to the number of layers containing heavier elements and their position is determined by the thickness and depth of these layers. Using this method it is possible to measure the layer parameters down to the thickness of about $10 \mathrm{~nm}$. The MCL technique allows one to obtain the depth of the luminescencing layer and to study the luminescence spectra from any layer of two-layer structures.

\section{Backscattering electron mode}

It is well known that the BSE signal from multilayer and thin film structures strongly depends on the primary electron energy $E_{\mathrm{b}}[4,5]$. Therefore, from the BSE signal dependence on $E_{\mathrm{b}}$ it is possible, in principle, to obtain such parameters of multilayer structures as their thickness and depth. To check this possibility the investigations of some test structures consisting of thin layers of aluminium and copper evaporated on silicon wafers were carried out. For measurements of the BSE signal either a semiconductor detector of backscattering electrons or an absorption current mode of the SEM with a negatively charged grid suppressing the secondary electron emission have been used. In the first case the signal is proportional to the backscattering electron energy, in the second one the backscattering coefficient can be obtained using the relation $\eta=\left(I_{\mathrm{b}}-I_{\mathrm{a}}\right) / I_{\mathrm{b}}$, where $I_{\mathrm{b}}$ and $I_{\mathrm{a}}$ are the beam and absorption current values, respectively.

The $E_{\mathrm{b}}$ dependences of the BSE coefficient and backscattering energy have maxima, a number of which depends on a number of copper layers and the maximum position depends on the depth and thickness of these layers (Fig. 1, 2). The contrast value $C=\left(S-S_{0}\right) / S_{0}$, where $S$ and $S_{0}$ are the BSE signals obtained on the structures discussed and silicon substrate, respectively. The left side of the $E_{\mathrm{b}}$ dependence can be approximately described as $E_{\mathrm{b}}^{0} \propto \sqrt{t_{\mathrm{Al}}}$, i.e. it is in good agreement with the energy dependence of the primary electron pulse relaxation length [1]. It should be also mentioned that there is the $E_{\mathrm{b}}$ range, where the BSE signal from the deeper copper layer is higher than from shallower one (Fig. 1b) which can be very useful for the characterization of multilayer metallization.

On the base of such measurements it is possible to develop non-destructive methods of characterization of the layer parameters for any particular structure. As it can be seen from Fig. 1a, this technique allows us to measure the parameters of the layers with thickness as small as $10 \mathrm{~nm}$. Calibration curves necessary for such measurements can be obtained from the investigations similar to those illustrated in Fig. 1 and 2 or by the Monte-Carlo simulation. 

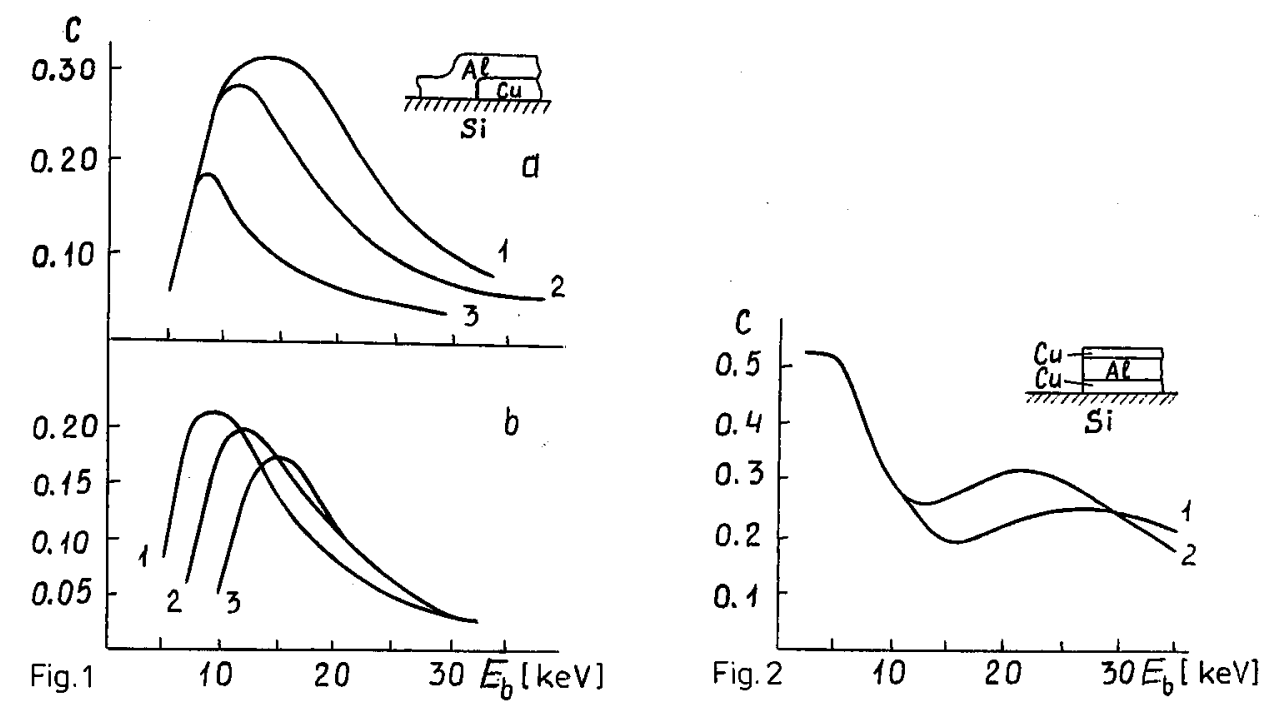

Fig. 1. Dependence of the BSE contrast on the primary electron energy $E_{\mathrm{b}}$ for the Si-Cu-Al structures with layer thicknesses: a) $t_{\mathrm{Al}}=160 \mathrm{~nm}, t_{\mathrm{Cu}}=180(1), 120$ (2), $60 \mathrm{~nm}(3)$; b) $t_{\mathrm{Al}}=100(1), 200(2), 300 \mathrm{~nm}(3), t_{\mathrm{Cu}_{\mathrm{u}}}=150 \mathrm{~nm}$.

Fig. 2. Dependence of the BSE contrast on the primary electron energy $E_{\mathrm{b}}$ for the $\mathrm{Si}-\mathrm{Cu}-\mathrm{Al}-\mathrm{Cu}$ structures, $t_{\mathrm{Al}}=450(1)$ and $300 \mathrm{~nm}(2), t_{\mathrm{Cu}}=100$ and $200 \mathrm{~nm}$ for the upper and lower layer, respectively.

\section{Modulated cathodoluminescence}

The cathodoluminescence (CL) mode is widely used for material characterization [6]. For the structures with layers parallel to the beam the spatial resolution of about $60 \mathrm{~nm}$ can be achieved in this mode [7]. But for the structures with layers perpendicular to the beam, which are more suitable for the non-destructive characterization, methods with the improved spatial resolution in depth should be developed. One of such techniques is discussed in the present section.

In the CL mode a signal from any point of a sample without a correction for a self-absorption, under low excitation conditions is proportional to:

$$
S_{\mathrm{CL}} \propto p(r)\left(\tau / \tau_{\mathrm{rr}}\right)=p(r)\left[\tau_{\mathrm{nr}} /\left(\tau_{\mathrm{nr}}+\tau_{\mathrm{rr}}\right)\right],
$$

where $p(r)$ is the minority carrier concentration in the point $r, \tau_{\mathrm{rr}}$ and $\tau_{\mathrm{nr}}$ are the excess carrier lifetimes determined by the radiative and nonradiative recombination, respectively. If the values of $\tau_{\mathrm{rr}}$ and $\tau_{\mathrm{nr}}$ are homogeneous inside each layer, the signal-depth dependence can be obtained from the $p(r)$ distribution. As a rule this distribution and therefore the CL signal-depth dependence can be described as a curve with a maximum at some depth. For example, such CL signal distribution takes place in the crystals with small diffusion lengths, where the depth dependence of the CL signal is very similar to that of electron-hole generation $h(z)$ which is a curve with a maximum. If the diffusion length is large enough a maximum in the CL signal-depth dependence can be stipulated by an existence of 
a "dead" layer near the surface associated with an enhanced surface recombination or with a depletion region formed near the surface due to charging of surface states. In any case, the position of this maximum depends on the electron-beam energy, the depth of the maximum increases with increasing $E_{\mathrm{b}}$ [8]. If the CL signal-depth distribution has a form of a curve with a maximum at some depth it is possible to separate the CL signals from different layers of a two-layer structure using the modulation of the primary electron energy $E_{\mathrm{b}}$ and studying the modulated CL signal [9].

To illustrate the basic principles of this technique let us assume that $E_{\mathrm{b}}$ is modulated with frequency $\omega$ as $E_{\mathrm{b}}=E_{0}\left(1+\Delta_{1} \sin \omega t\right)$ and the electron beam current $I_{\mathrm{b}}$ is modulated as $I_{\mathrm{b}}=I_{0}\left(1+\Delta_{2} \sin \omega t\right)$, where $\Delta_{1}$ and $\Delta_{2}$ are the modulation amplitudes. In this case the CL signal measured at wavelength $\lambda$ is given by

$$
\begin{aligned}
S_{\lambda}= & B E_{0} I_{0} \int_{-\infty}^{\infty} \int_{-\infty}^{\infty} \int_{0}^{\infty}\left(1+\Delta_{1} \sin \omega t\right)\left(1+\Delta_{2} \sin \omega t\right) A_{\lambda}(z) \\
& \times p(x, y, z) \mathrm{d} x \mathrm{~d} y \mathrm{~d} z=B E_{0} I_{0} \int_{0}^{\infty} A_{\lambda}(z) f\left(E_{0}, \Delta_{1}, \Delta_{2}, \omega, z\right) \mathrm{d} z,
\end{aligned}
$$

where $A_{\lambda}$ is the coefficient determined by the concentration of radiative recombination centers and by the luminescence efficiency of these centers, $B$ is the coefficient dependent on geometric factors and on the experimental set-up used and not associated with luminescence properties. The MCL signal measured at frequency $\omega$ for the two-layer structure, assuming that $A_{\lambda}$ is constant inside each layer, is:

$$
S_{\lambda, \omega}=B E_{0} I_{0}\left(A_{1} \int_{0}^{t_{1}} f_{\omega} \mathrm{d} z+A_{2} \int_{t 1}^{\infty} f_{\omega} \mathrm{d} z\right)=I_{1 \omega}+I_{2 \omega},
$$

where $t_{1}$ is the upper layer thickness, $f_{\omega}$ is the first-order term of the $f$ harmonic expansion. A general view of $f_{\omega}$ calculated under the assumptions that $p\left(E_{\mathrm{b}}, z\right)$ is proportional to the depth generation distribution $h\left(E_{\mathrm{b}}, z\right)$, i.e. that the diffusion length in the both layers is very small, and that $h\left(E_{\mathrm{b}}, z\right)$ can be approximated by the expression given in [10], is presented in Fig. 3 . It is seen that $f_{\omega}$ changes a sign at some depth $z_{0}$ depending on $E_{0}, \Delta_{1}$ and $\Delta_{2}$ values. Therefore, if only $E_{\mathrm{b}}$ is modulated $\left(\Delta_{2}=0\right)$ it is possible, changing the $E_{0}$ value, to achieve the situation when either $I_{1 \omega}$ or $I_{2 \omega}$ integral is equal to zero, i.e. when the MCL signal is associated with only one particular layer [9]. This allows us to study the CL spectra from any of two layers or to obtain the thickness of the upper layer, from the measurements of the MCL signal dependence on $E_{\mathrm{b}}$ if the CL spectra are known. Using the electron-beam intensity modulation provides some additional possibilities, e.g. $I_{1 \omega}$ or $I_{2 \omega}$ integrals can be made equal to zero at constant $E_{0}$ only by choosing the appropriate $\Delta_{2}$ value.

The experiments have been carried out on the two-layer structure consisting of $\mathrm{ZnS}$ single crystal with deposited on it $\mathrm{ZnS}: \mathrm{Mn}$ layer with a thickness of about $2 \mu \mathrm{m}$. To modulate the electron-beam energy an ac voltage was applied to the sample stage, i.e. $E_{\mathrm{b}}=E_{0}\left(1+\Delta_{1} \sin \omega t\right)$, where $\omega / 2 \pi=340 \mathrm{~Hz}, E_{0 \max }=30 \mathrm{keV}$. The beam current was modulated as $I_{\mathrm{b}}=I_{0}\left(1+\Delta_{2} \sin \omega t\right)$. The measurements of the MCL signal were carried out at modulation frequency using a lock-in amplifier. 


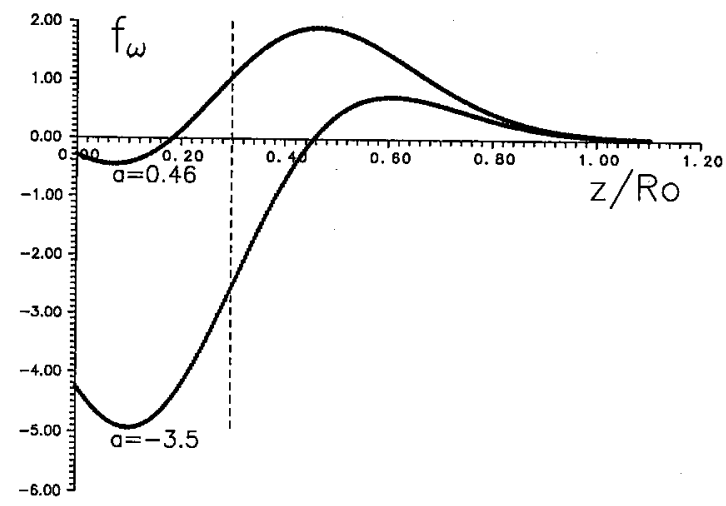

Fig. 3. Dependence of $f_{\omega}$ function on $z / R_{0}$, where $R_{0}$ is the electron penetration depth for two values of $a=\Delta_{2} / \Delta_{1}, t_{1}=0.3 R_{0}$.

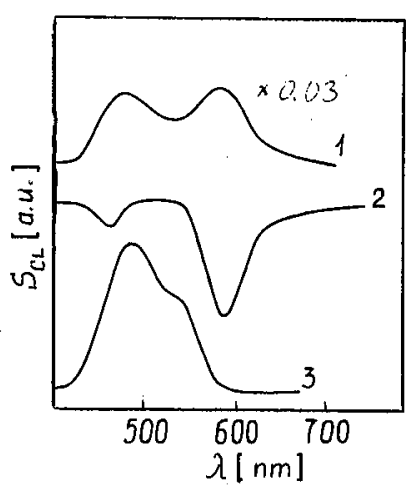

Fig. 4. CL spectra measured on $\mathrm{ZnS}-\mathrm{ZnS}: \mathrm{Mn}$ structures without (1) and with the energy and beam current modulation $(2,3)$. The CL spectra from the upper (2) and lower (3) layers can be separated.

The results obtained are presented in Fig. 4. The curve 1 is the CL spectrum obtained without any modulation of the beam intensity and voltage. When using the energy modulation only at $E_{0}=30 \mathrm{keV}$ the signal from the $\mathrm{ZnS}$ layer (lower layer) is positive, the signal from the upper one is negative. With decreasing $E_{0}$ to $27 \mathrm{keV}$ the signal from the upper layer increases up to zero and with further decreasing of $E_{0}$ it inverts the sign from negative to positive one. The signal from the lower layer at all beam energies is positive. This evidences that in our case the maximum of the $p\left(E_{\mathrm{b}}, z\right)$ dependence is in the upper layer at all energies used. When using both energy and intensity modulation, the intensity modulation being in antiphase with the energy modulation $\left(\Delta_{1}>0, \Delta_{2}<0\right)$ at $E_{0}=30 \mathrm{keV}$, it is possible, choosing the $\Delta_{2}$ value, (see Fig. 3 , lower curve) to decrease the signal from the lower layer to zero and the MCL spectrum obtained under these conditions 
is associated with the upper $\mathrm{ZnS}: \mathrm{Mn}$ layer (Fig. 4, curve 2). If $\Delta_{2}>0$ it is possible to compensate the signal from the upper layer (Fig. 3, upper curve) and to measure the CL spectrum from the lower layer (Fig. 4, curve 3). Our estimations have shown that for some structures this technique allows us to achieve a spatial resolution in depth in the range of $10-100 \mathrm{~nm}$.

\section{Conclusions}

The experiments carried out have shown that the methods discussed are very promising for the non-destructive characterization of planar multilayer structures. These techniques provide a high spatial resolution in measurements of the depth distribution of structure parameters. To use these techniques for measurements of dimensions and physical properties of multilayer structures it is necessary to develop the methods of the signal computer simulation and depth profile reconstruction. The spatial resolution in depth of the both techniques can achieve the value of about $10 \mathrm{~nm}$.

\section{References}

[1] V.V. Aristov, V.V. Kazmiruk, N.G. Ushakov, E.B. Yakimov, S.I. Zaitsev, Vacuum 38, 1045 (1988).

[2] V.V. Aristov, N.N. Dryomova, A.A. Firsova, V.V. Kazmiruk, A.V. Samsonovich, N.G. Ushakov, S.I. Zaitsev, Scanning 13, 15 (1991).

[3] E. Yakimov, Scanning Microsc. 6, 81 (1992).

[4] H. Niedrig, J. Appl. Phys. 53, R15 (1982).

[5] V.V. Aristov, N.N. Dryomova, S.K. Likharev, E.A. Rau, Electronnaya promyshlennost 2, 44 (1990) (in Russian).

[6] D.B. Holt, B.G. Yakobi, in: SEM Microcharacterizalion of Semiconductors, Eds. D.B. Holt, D.C. Joy, Academic Press, London 1989, p. 372.

[7] C.A. Warwick, J. Phys. IV 1, C6-117 (1991).

[8] W. Hergert, L. Pasemann, Phys. Status Solidi A 85, 641 (1984).

[9] V.A. Kireev, I.I. Razgonov, Zh. Tekh. Fiz. 59, No 4, 180 (1989).

[10] C.J. Wu, D.B. Wittry, J. Appl. Phys. 49, 2827 (1978). 